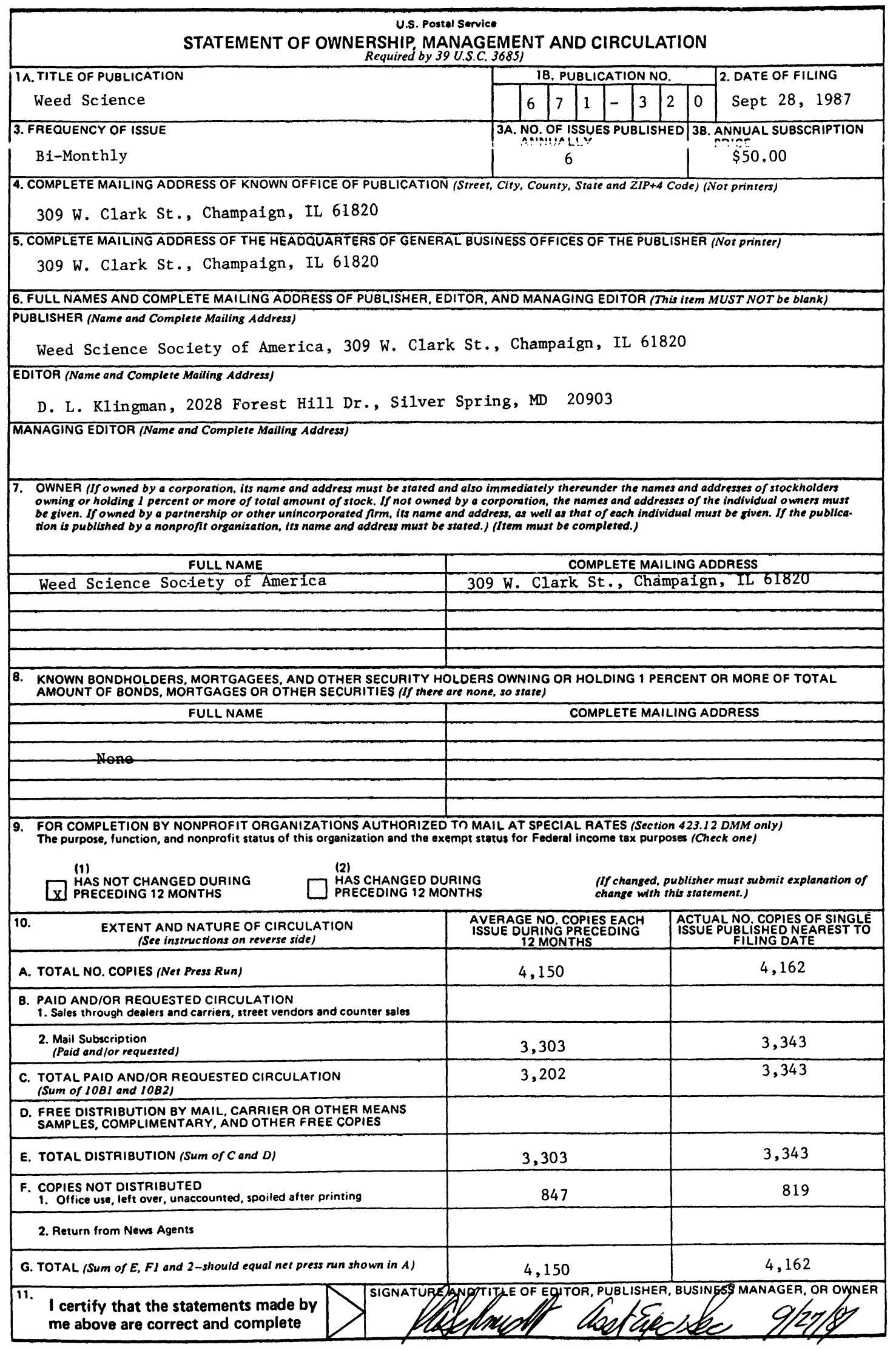




\section{Copies of articles}

from this publication are now available from the UMI Article Clearinghouse.

For more information about the Clearinghouse, please fill out and mail back the coupon below.

Yes! I would like to know more about UMI Article Clearinghouse.

I am interested in electronic ordering through the following

system(s):
$\square$ DIALOG/Dialorder
$\square$ OnTyme
$\square$ ITT Dialcom
$\square$ OCLC ILL Subsystem
$\square$ Other (please specify)
$\square$ I am interested in sending my order by mail.
$\square$ Please send me your current catalog and user instructions for the system(s) I checked above.

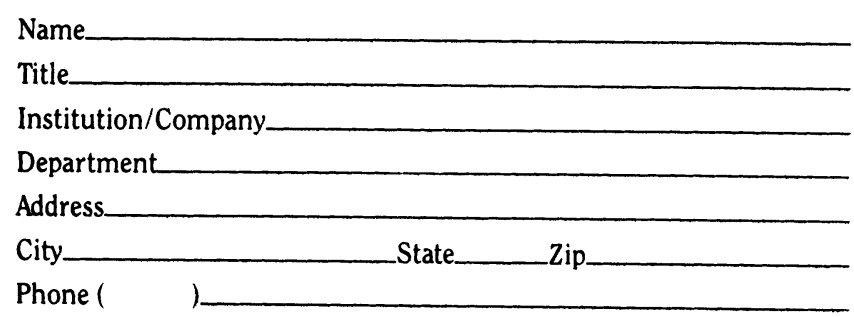

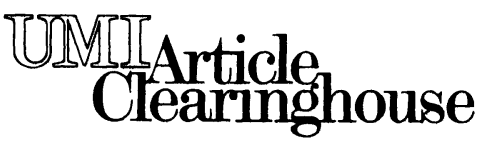

Mail to:

University Microfilms International

300 North Zeeb Road, Box 91 Ann Arbor, MI 48106 


\section{Common and Chemical Names of Herbicides a}

Common Name or Designation

acetochlor (ə-se-t.s-klōr) acifluorfen (as-e-floor-fen) acrolein ( $\cdot \cdot k r \bar{o}-\bar{l} \bar{e}-2 n)$ alachlor ('al-a-klor) ametryn (am-a-trin) amitrole ('am-a-tról) AMS

asulam ('az-u-lam)

atrazine ('a-tra-zēn)

barban (bär-ban) benefin ('ben-ə-fin) bensulfuron (ben-sul-fü-ron) bensulide ( bentazon ('ben-ta-zon) benzofluor ('ben-zō-flōōr) benzoylprop ('ben-zô-il-prop) bifenox ("bi-fen-oks) bromacil ('brom-a sil) bromoxynil (brom-'oks-i-nil) butachlor ( $b u$-ta-klor) buthidazole (bu- thid-a-zol) butylate ('bü-ti-lāat)

cacodylic acid ( $\mathrm{ka}-\mathrm{ko}-{ }^{-} \mathrm{dil}-\mathrm{ik}$ as-id) CDAA

chloramben (kiorr-am-ben) chlorimuron (klo-rim- $-\mathbf{u}-r o n)$ chloroxuron (klōr- oks-u-ton) chlorpropham (klör- prōfam) chlorsulfuron (klōr- 'sul-fü-ron) cinmethylin (sin- meth-o-lin) clethodim ('kleth-o-dim) clomazone ( $k J \bar{o}-\mathrm{ma}$-zone) cloproxydim (klo-proks-a-dim) clopyralid (klo-pir-a-lid) CMA

cyanazine (si- an-a-zēn) cycloate ('si-klö-àt)

dalapon ('dal-a-pon) dazomet ('daz-ō-met) DCPA

desmedipham (dez-'med-a-fam) diallate ('di-a-liat)

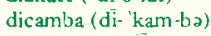
dichlobenil (di-klo- ben-al) dichlorprop ('di-klör-prop) diclofop ('di-klo-fop) diethatyl (di-eth-ə-til) difenzoquat (di- fen-zo-kwät) dinitramine (di- 'nit-ra-mēn) dinoseb ('di-nō-seb) diphenamid (di- fen-a-mid) dipropetryn (di- pröp-a-trin)

diquat ('di-kwat)

diuron ('di-u-ron)

DSMA

endothall (en-do-thol)

EPTC

ethalfluralin (eth-əl- flur-ə-lin) ethofumesate (eth-ö- fü-ma-sat)

fenac (fen-ak)

fenoxaprop (fen-oks-a-prop)

fenuron ('fen- $\overline{\mathbf{u}}$-ron)

fenuron TCA (fen-ī-ron)

flamprop (nam-prop)

fluazifop (floo- az-i-fop)

fluazifop-P (flü- az-i-fäp-P)

fluchloralin (flōo-klor-z-lin)

fluometuron (floo-o- met-ij-ron)

fluorochloridone (floor-o- ktor-2-don)

fluoroglycofen (flü(-a)r-ó-gli-ka-fən)

fluridone ('flur-i-dōn)

fornesafen (fo-'mes-ə-fen)

fosamine ( fos-3-mēn)

glyphosate ('gli-fō-sāt)

haloxy fop (ha- loks-e-fop) hexaflurate (heks-ว-'flur-āt) hexazinone (heks- àz-ว-nön)

\section{Chemical Name}

2-chloro- 2 -(ethoxymethyl)-X-(2-ethyl-6-methylphenyl) acetamidc

5-[2-chloro-4-(trifluoromethyl)phenoxy]-2-nitrobenzoic acid

2-propenal

2 -chloro-X-(2,6-dicthylphenyl)-V-(methoxymethyl)acetanide

V-ethyl-1"-(1-methylethyl)-6-(methylthio)-1,3,5-triazine-2,4-dianine

$1 / 1-1,2,4$-triazol-3-anine

ammonium sulfamate

metholl(t-aminophenyl)sulfonyll carbamate

6-chloro-1 ethy - ${ }^{\prime \prime}$ (1-methylethvl)-1,3,5-triazine-2,4-diamine

4-chloro-2-butynyl 3-chlorophenylearbamate

$\lambda$ butyl-N-ethyl-2,6-dinitro-4-(trifluorome thyl)benzenanine

2-[ [| [|4,6-dimethoxy-2-pyrimidinyl) aninol carbonyl] amino] sulfonyl| methyl] benzoic acid

O,O-bis(1-methylethyl) $\mathrm{S} \cdot \mid 2 \cdot[$ (phenvlsulfonyl)aminol ethyl] phosphorodithioate

3-(1-methylethyl)-(1II)-2,1,3-benzothiadiazin-4(3H)-one 2.2-dioxide

1-[4-(ethylthio)-2-(trifluoromethyl)phenyl] methanesulfonamide

$\checkmark$-benzoyl- $N-(3,+$-dichlorophenyl)- $-L-$-alanine

methyl 5-(2,4-dichlorophenoxy)-2-nitrobenzoate

5-bromo-6-methyl-3-(1-methylpropyl)-2, $+(1 H, 3 H)$ pyrimidinedione

3,5-dibromo-thwdroxvbenzonjtrile

$N$-(buthoxymethyl)-2-chloro- $N$-(2,6-diethylphenvl)acetamide

3-|5-(1,1-dimethylethyl)-1,3,4-thiadiazol-2-y||-4-hydroxy-1-methyl-2-imidazolidinone

S-ethyl bis(2-methylpropyl)carbamothioate

dimethyl arsinic acid

2-chloro-V-di-2-propenvlacetamide

3 -amino-2,5-tichlorobenzoic acid

2-[ [I[4-chloro-6-methoxy-2-pyrimidiny]) amino] carbonyll aminol sulfonyl] benzoic acid

V'-14-(4-chiorophenoxy)phenyl]-N, $\lambda$-dimethylurea

1 -methylethyl 3-chlorophenylcarbamate

2-chloro-X-( $($ (4-methoxy-6-methyl-1,3,5-triazin-2-yl)amino $\}$ carbonyl] benzenesulfonamide

exo-1-methyl-4-(1-methylethyl)-2-\{(2-methylphenyl)methoxyl-7-oxabicyclol 2.2.1) heptane

(t,L)-(土)-2-|1-||(3-chloro-2-propenyl)oxyl iminol propyl]-5-|2-(cthylthio)propyl|-3-hydroxy-2-cyclohenen-1-one

2-f(2-chlorophenyl) methyll-4.4-dimethyl-3-isoxazolidinone

(L, E)-2-[1-[|(3-chloro-2-propenyl)oxy] iminol buty]l-5-|2-(cthylthio)propyll-3-hydroxy-2-cylcohexen-1-one

3,6-dichloro-2-pyridinecarboxylic acid

calcium salt of MAN

2-|14-chloro-6-(ethylamino)-1,3.5-triazin-2-y\| aminol-2-methylpropanenitrile

S-ethyl cyclohexylethylcarbamothioate

2,2-dichloropropanoic acid

tetrahydro-3,5-dimethyl-2JI-1,3,5-thiadiazine-2-thione

dimethyl 2,3,5,6-tetrachloro-1,4-benzenedicarboxylate

ethy] [3-i[(phenylamino) carbonyl| oxy phenvl| carbamate

S-(2,3-dichloro-2-propeny]) bis(1-methylethyl)earbanothioate

3,6-dichloro-2-methoxybenzoic acid

2,6-dichlorobenzonitrile

$( \pm)-2-(2,+$-dichlorophenoxy $)$ propanoic acid

( \pm -2-[4-(2.4-dichlorophenoxy)phenoxylpropanoic acid

$\forall$-(chloroacervl) $-(2,6$-diethylphenyl)glycine

1,2-dimethyl-3,5-diphenyl-1H-pyrazolium

$v^{3} v^{3}$-diethyl-2,4-dinitro- 6 -(trifluoromethyl)-1,3-benzenediamine

2-(1-methylpropyl)-4.6-dinitrophenol

$\therefore$ - dimethyl- $\alpha$-phenyl benzeneacctam ide

6-(ethylthio)-N, $\lambda^{\prime}$-bis(1-methylethyl)-1,3,5-triazine-2,4-diamine

6.7-dihydrodipyrido $\left|1,2-\alpha: 2^{\prime}, 1^{\prime}-c\right|$ pyrazinedium ion

$\mathrm{N}$ '-(3,4-dichloroplenyl)-1.1-dimethylurea

disodium salt of MAA

7-oxabicyclol 2.2.11 heptane-2,3-dicarboxylic acid

$S$-ethvl dipropylcarbamothioate

$\checkmark$-ethyl-V-(2-methyl-2-propenyl)-2,6-dinitro-4-(trifluoromethyl)benzenamin

( \pm -2-ethoxy-2,3-dihydro-3,3-dimethyl-5-benzofuranyl methanesulfonate

2,3,6-trichlorobenzeneacetic acid

(士)-2-[4-[(6-chloro-2-benzoxazolyl)oxy] phenoxy] propanoic acid

N.V-dimethyl: phenylure.

Salt of fenuron and $\mathrm{TCA}$

$\checkmark$-benzoyl- $-(3$-chloro-4-fluorophenyl)- 1$) I$-alanine

(+)-2-|4-|| 5-(trifluoromethy|)-2-pyridinyl] oxy | phenoxyl propanoic acid

(K)-2-|4-||5-(trifluoromethyl)-2-pyridinyl| oxyl phenoxy/ propanoic acid

N-(2-chloroethyl)-2,6-dinitro-i'-propyl-4-(trifluoromethyl)benzenamine

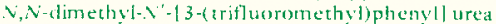

3-chloro-4-(chloromethyl)-1-13-(triflueromethyl)phenyli-2-pyrrolidinone

carboxymethyl 5-[2-chloro-4-(trifluorome thyl)phenoxy]-2-nitrobenzod te

1 -methyl-3-phenyl-5 [3-(trifluoromed $\left.]_{1 y}\right)$ phenyl|-4(1H)-pyridinone

5-[2-chloro-4-(trifluronethyl)phenoxy|-1 (methylsul fonyl)-2-nitrobenzamide

ethyl hydrogen (aminocarbonvi)phosphonate

N-(phosphonomethyl)glycine

2-|4-||3-chloro-5-(trifluoromethy!)-2-pyridinyll oxy| phenoxy| propanoic acid potassium hexafluoroarsenatc

3-cyclohexyl-6-(dimethylamino)-1-metlyl-1,3.5-triazine-2,4(1/H,3/I)-dinne

(1)-2-1 4,5-dihydro-4-methyl-4-(1-methyle thyl)-5-0xo-1//-im diazol-2-yl] -3-pyridinecarloxylic acid $2 \cdot[4,5$-dihydro-4methyl-4-(1-methylethyl)-5-oxo-1H/-imidazol-2-yl|-3-quinolinccarboxvlic acid

PRONUNCIATION KEY: āpe, fat, cär, bâre; êvil, ten; bite, is; gō, lot, oil, out, tōol, look; cūre, cup, tuғn; ə = a in ago. 


\section{Common Name or Designation}

imazethapyr (im-a-'zeth-o-pir)
ioxynil (i- oks-i-nil)

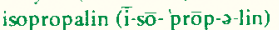

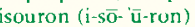
isoxaben (i-soks-a-ben)

karbutilate (kär- 'bū-tə-lāt)

lactofen ('lak-to-fen)

linuron (lin-ü-ron)

MAA

MAMA

MCPA

$\mathrm{MCPB}$

mecoprop ('mek-o-prop)

mefluidide (me- flōo-i-did)

metham ('meth-am)

methazole ('meth-ว-zo])

metolachlor (ma-tol-a-klor)

metribuzin ('me-tri- būi-zəan)

metsulfuron (met-sul-fü-ron)

MH

molinate ( mō-lin-āt)

monuron (mon-ü-ron)

monuron 'I'CA (mon-u-ron)

napropamide (na-pro-ps-mid) naptalam ('nap-ta-lam)

nitrofen ('ni-tro-fen)

norea (nor- $\bar{c}-a$ )

norflurazon (nôr- flur-a-zon)

oryzalin (ō- ríz-o-lin)

oxadiazon (oks-a-di-a-zon)

oxyfluorfen (okse. flōor-fen)

paraquat ( pâr-ə-kwit)

pebulate (peb-u-lat)

pendimethalin (pen-di-meth-a-lin)

perfluidone (pur- floo-i-don)

phenmedipham (fen-'med-a-fam)

picloram ( pik-lō-ram)

profluralin (prō-'flur-a-liii)

prometon ('prö-ma-ton)

prometryn ( prō-ma-trin)

pronamide (pro-na-mid)

propachlor ( prō-pa-klor)

propanil ( prō-po-nil)

propazine (pró-pa-zen)

propham ( prō-fam)

pyrazon ( pi-ro-zon)

quizalofop (quiz-aj-o-fop)

sethoxvdim (seth-oks-ci-din))

siduron ('sid- $\bar{u}-r o n$ )

simazine ('sim-ə-zēn)

sulfometuron (sul-fó-'met-ū-ron)

TCA

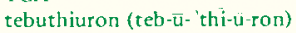

terbacil ('tur-ba-sil)

terbutrun ( $\mathrm{tur}-\mathrm{b} \overline{\mathrm{u}}-\mathrm{trin})$

thiameturon (thi-a-met-ü-ron)

thiobencarb (thi-o- ben-kärb)

triallate ('tri-a-lat)

triclopyr ('tri-klo-pir)

tridiphane ( tri-di-făn)

trifluralin (tri-'fler-a-lin

2,4-D

$2,4-\mathrm{DB}$

vernol ate ('vur-nōlat)

xylachlor ( $z \bar{i}-\mid a-k l o r)$
Chemical Name

( \pm )-2-14,5-dihydro-4-methyl-4-(1-methylethyl)-5-oxo-1H-imidazol-2-y!l-5-ethyl-3-pyridinecarboxylic acid

4-hydroxy-3,5-diiodobenzonitrile

4-(1-methylethyl)-2,6-dinitro- $N, N$-dipropylbenzenamine

$N^{\prime}$-[5-(1,1-dimethylethyl)-3-isoxazolyl]-N,N-dimethylurea

$\mathrm{N}$-[3-(1-ethy]-1-methylpropy])-5-isoxazoly1]-2,6-dimethoxybenzamide

3-[ [(dimethylamino)carbonyl] amino] phenyl (1,1-dimethylethyl)carbamate

(t)-2-ethoxy-1-methyl-2-oxoethyl 5-[2-chloro-4-(trifluoromethyl)phenoxy]-2-nitrobenzoate

$N^{\prime}-(3,4$-dichlorophenyl $)-N$-methoxy- $N$-methylurea

methylarsonic acid

monoammonium salt of MAA

(4-chloro-2-methylphenoxy)acetic acid

4-(4-chloro-2-methylphenoxy)butanoic acid

(4)-2-(4-chloro-2-methylphenoxy)propanoic acid

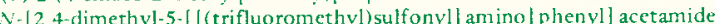

methylcarbamodithioic acid

2-(3.4-dichlorophenyl)-4-methyl-1,2,4-oxadiazolidine-3,5-dione

2-chloro- $\mathrm{N}$-(2-ethyl-6-methylphenyl)-N-(2-methoxy-1-methylethyl)acetamide

4-amino-6-(1,1-dimethylethyl)-3-(methylthio)-1,2,4-triazin-5(4H)-one

2-[|| (4-methoxy-6-methyl-1.3,5-triazin-2-yl) aminol carbonyl amino] sulfonyl] bcnzoic acid

1. 2-dihydro-3.6-pyridazinedione

S-ethyl hexahydro-1/l-azepine-1-carbothioate

$N^{\prime}$-(4-chlorophenyl)-N,N-dime thylu rea

Salt of monuron and TCA

monusudium salt of $M A$

V.. -diethyl-2-(1-naphthalenyloxy)propanamide

$2 \cdot \mid(1$-naphthalenylamino) arbonyl| benzoic acid

2.4-dichloro-1-(4-nitrophenoxy)benzene

X.dimethyl- "(octahydro-4,7-methano- $1 \mathrm{H}$-inden-5-yl)urea $3 \mathrm{a} \alpha, 4 \alpha, 5 \alpha, 7 \alpha, 7 \mathrm{a} \alpha$-isomer

+-chloro-5-(methylamino)-2-(3-(trifluoromethyl)phenyl)-3(2lI)-pyridazinone

4-(dipropylam ino)-3,5-dinitrobenzenesulfonamide

3-(2,4-dichloro-5-(1-methylethoxy)phenv! -5-(1.1-dimetly ylethyl)-1,3.4-oxadiazol-2-(3/I)-ono

2-chloro-1-(3-cthoxy-4-nitrophenoxy)-4(trifluoromethyl)benzenc

$1,1^{\prime}$-dinethyl-4,4'-bipyridinium ion

S-propyl butylethylcarbamothioate

(1-ethylpropyl)-3.4-dimethyl-2,6-dinitrobenzenanine

1,1.1-trifluoro-i-[2-methyl-t-(pheny]sulfonyl)phenyl| methanesulfonamide

3-1(nethoxycarbonyt)amino phenyl (3-methylphenyl) arbamate

4-amino-3,5.6-trichloro-2-pyridinecarboxylic acid

$\mathrm{N}$-(cyclopropylmethyl)-2,6-dinitro-N-propyl-4-(trifluoromethyl)benzenamine

6-methoxy-N.N"bis(1-methylethyl)-1.3,5-triazine-2,4-diamine

$\therefore . N^{\prime}$-bis 1 -methylethyl)-6-(methylthio)-1,3,5-triacine-2,4-diamine

3.5-1ichloro( $1-1,1$-dimethyl-2-propynyl)benzamide

2-chloro- - -(1-methylethyl) $N$-phenvlacetamide

$\checkmark(3,4$-dichlorophenyl)propanamide

6 -chloro- $N, N^{\prime \prime}$-bis 1 -methylethyl)-1,3,5-triazine-2,4-diamino

1 -methylethyl phenylcarbanate

5-amino-4-chloro-2-phenyl-3(2H)-pyridazinone

(+)-2-|4|(6-chloro-2-quinoxalinyl)oxyl phenoxy| propanoic acid

2-[1-(etroxyimino)butyl] -5-[2-(ethylthio)propy]]-3-hydroxy-2-cyclohexen-1-one

$\mathrm{N}-(2 \cdot$ methylcyclohe $y \mathrm{l})-\mathrm{N}$-phenylurea

6-chloro-i, N'-diethyl-1,3,5-triazine-2,4-diamine

2-[ [ [ [ (4,6-dimethy]-2-pyrimidiny!) amino/ carbonyl] amino] sulfonyl] benzoic acid

trichloroacetic acid

-[5-(1,1-dimethylethyl)-1,3,4-thiadiazol-2-yl]-N,N'-dimethylurea

5-chloro-3-(1.1-dimethylethyl)-6-mcthvl-2,4(1H,3H)-pyrimidinedione

$\mathcal{N}$-(1,1-dimethylethyl) $N$ '-ethyl-6-(methylthio)-1,3,5-triazine-2,4-diamine

3-|[|[(4-methoxy-6-methy]-1,3,5-triazin-2-yl) aminol carbonyll aminol sulfony!l-2

thiophenecarboxylic acid

$S$ - (t-chlorophenyi)methy] $]$ dethylcarbamothioate

\$-(2,3,3-trichloro-2-propenyl) bis(1-methylethyl)carbamothioate

[(3,5.6-trichloro-2-pyridinyl)oxy] acetic acid

2-(3.5-dichlorophenyl)-2-(2.2.2-trichluroethyl)oxirane

2,6-dinitro-N. $\mathrm{A}^{-}$-dipropyl-4-(trifluoromethyl)benzenamine

(2,4-dichlorophenoxy)acetic acid

4-(2,4-dichlorophenoxy)butanoic acid

S-propyl dipropylcarbamothioate

2-chloro-N-(2,3-dimethylphenyl)-N-(1-methylethyl)acetamide

Common and Chemical Names of Herbicide Modifiers cyometrinil (si-̄o-'met-ri-nil)

dichlormid ('di-klōr-mid)

dietholate ('di-eth-o-lat)

flurazole (flur-ə-zol)

mephenate ('mef-a-nāt)
(Z)- $\alpha$ [(cyanomethoxy) iminol benzeneacetonitrile

2,2-dichloro- $N, N$-di-2-propenylacetamide

$O, O$-diethyl $O$-phenyl phosphorothioate

phenylmethyl 2-chloro-4-(trifluoromethyl)-5-thiazolecarboxylate

4-chlorophenyl methylcarbamate

${ }^{\mathrm{a}} \mathrm{Herbicides}$ no longer in use in USA are omitted. Complete listing, including these, is in WEEDS 14(4), 1966 and in Weed Science $23(1), 1975$. 